\title{
Non-linear MHD convective flow of Carreau nanofluid over an exponentially stretching surface with activation energy and viscous dissipation
}

\author{
K. S. Srinivasa Babu' ${ }^{1,2} \cdot$ A. Parandhama ${ }^{3} \cdot$ R. Bhuvana Vijaya ${ }^{1}$
}

Received: 12 September 2020 / Accepted: 4 February 2021 / Published online: 27 February 2021

(c) The Author(s) 2021 OPEN

\begin{abstract}
Numerical approach for a non-linear mixed convective magnetohydrodynamic two-dimensional Carreau nanofluid through an exponentially permeable stretching surface with viscous dissipation and velocity slip under the influence of Arrhenius activation energy in chemical reaction is reported. The effects of thermophoresis and Brownian motion are considered. The governing nonlinear equations of this model are transmuted into ODE's through similarity variables and solved them with a shooting method based on R-K 4th order. Responses of fluid velocity, transfer rates (heat and mass) versus pertinent parameters of the problem for suitable values are obtained and the computational calculations for friction coefficient, Nusselt number and Sherwood number for the both suction and injections regions are presented in plots and tables. It is found that fluid velocity is an increasing function of Weissenberg number. Momentum boundary layer thickness is depressed by magnetic field impact. Increasing trend in Carreau fluid temperature is noticed due to larger values of thermophoresis and Brownian motion effects. Concentration field is a decreasing function of Brownian motion but an increasing function of thermophoresis. Activation energy augments the concentration curves and lowered by Schmidt number. Comparison of the results is made with already published results and we got good agreement.
\end{abstract}

Keywords Nonlinear mixed convection · MHD · Exponential stretching · Carreau nanofluid · Activation energy

\section{Introduction}

The studies of boundary layer flow and transfer of heat and mass energies over stretching and/or shrinking surfaces are extensively presented by many researchers due to their vast technological applications such as design of electronic chips, preparation of metallic and plastic sheets. The basic work on those boundaries is made by Crane [1]. Later several investigations done to study the behaviors of flow and heat and mass transfer over linear, non-linear, stretching surfaces with variable thickness, exponential stretching surfaces etc. Few of such studies are mentioned here. Khedr et al. [2] presented the characteristics of MHD flow of a micropolar fluid past a stretching permeable surface along with heat generation or absorption. An analytical investigation is made by Magyari and Chamkha [3] to discuss the combined effect of heat generation or absorption and first-order chemical reaction on micropolar fluid flow via a permeable stretched surface. Seyadi et al. [4] studied numerically the behavior of heat and mass transfer of MHD Eyring-Powell flow in a stretching channel along with the effects of radiation, chemical reaction and heat generation/absorption. They used a high accuracy numerical method based on the Galerkin method with Multi-wavelet basis (MWGM) to obtain the solutions of coupled differential equations. A similar investigation is

K. S. Srinivasa Babu, ssbkasibhotla@gmail.com | 1 Department of Mathematics, JNTUA College of Engineering, Ananthapuramu 515002 , India. ${ }^{2}$ Department of EM\&H, S.R.K.R. Engineering College, Bhimavaram 534 204, India. ${ }^{3}$ Department of Mathematics, Sree Vidyanikethan Engineering College, Tirupati 517 102, India. 
reported to study the combined effect of heat generation or absorption and first-order chemical reaction on natural convective micropolar fluid flow past a uniformly stretching permeable surface by Damseh et al. [5]. Takhar and Chamkha [6] studied the unsteady laminar boundary-layer flow of a viscous electrically conducting motion of a fluid over an impulsive stretching of a flat surface numerically by using implicit finite-difference scheme. Swati Mukhopadhyay [7] discussed the velocity and thermal slip effects on MHD boundary layer flow towards a porous exponentially stretching sheet with suction/blowing and thermal radiation. Hsiao [8, 9] discussed the behavior of nanofluids over stretching surfaces with different physical aspects.

In recent years, developing tendency in nanotechnology and nanoscience due to their importance in the fields of science and engineering that reveals synthesis and progressions of distinct nanomaterials. Enormous studies on nanomaterials and nanofluids have been carried by many scientists and engineers. Nanofluids are attracted by several researchers due to their absorbing and thermal transport behaviors in distinct fields. As nanofluids have high thermal conductivity, they got constancy to prevent rapid settling and obstacle besides the boundaries of heat transmission regions. Involvement of nanofluids happen in micro-manufacturing processes, cells of fuel, cooling processes, chips of microprocessor designs and their usage, nuclear industries, the apparatus of chemical and mechanical fields and in several designs of devices etc. The introduction of nanoparticles is made through Choi [10]. He depicted that thermal properties of fluid is improved by the involvement of nanoparticles. Main advantage noted in the usage of nanoparticles is that they able to work to gain specific determinations like the decline of friction, the heat transfer coefficient and thermal energy storage. The convective transport phenomena for nanofluids is discussed by Buongiorno [11] through a mathematical model in which nanofluid flow comprising of Brownian motion and thermophoretic dispersion of the nanoparticles is presented. In literature so much of noteworthy works are found on nanofluids by the engineers and scientists. Daniel et al. [12] studied the thermophoretic and Brownian motion effects of stratified electro magnetohydrodynamic flow under convective heat and mass conditions. Hayat et al. [13] discussed the characteristics of Carreau fluid flow and the heat transfer over a permeable stretched sheet using Homotopy method. Reddy and Sandeep [14] have jointly worked on the Carreau liquid flow and transfer of heat with cross diffusion effects via a permeable stretchable surface along with convective slip boundaries and thermal radiation. With the help of Cattaneo-Christov heat flux modelling, Ramadevi et al. [15] reported the magnetohydrodynamic flow corresponding to Carreau fluid over a melting surface. They discussed the results for Newtonian fluid and non-Newtonian fluid behaviors in their study. Two-dimensional magnetohydrodynamic flow of Carreau liquid through a variable stretched sheet is an investigation of Khan et al. [16]. Farroq et al. [17] also obtained the local similarity solutions for Carreau stagnation point flow past a non-linear stretched surface along with double stratification effects. A numerical work also due to homogeneous-heterogeneous reactions of Carreau fluid flow through stretchable cylinder has been discussed by Khan et al. [18]. Eid et al. [19] reported a numerical work on Carreau nanofluid flow over the permeable nonlinear stretchable surface. A numerical analysis due to Arrhenius activation energy along with binary chemical reaction for a magnetohydrodynamic Carreau fluid flow under Cattaneo-Christov heat flux model is a study of Kumar et al. [20]. Sulochana et al. [21] reported a Stagnation-point Carreau nanofluid flow past a permeable surface with transpiration effect, thermophoresis and Brownian motion. A non-Darcy magnetohydrodynamic Carreau fluid flow over permeable stretching surface is another similar investigation by Kala et al. [22]. Mamatha et al. [23] depicted the impact of convective boundary conditions applied for Carreau magnetohydrodynamic dusty fluid under stretching surface with a heat source. Waqas et al. [24] discussed numerically the effects of Brownian motion and thermophoresis on magnetohydrodynamic stretchable flow of convected Carreau nanoliquid over an exponentially stretching surface. Khan et al. [25] presented the unsteady heat and mass transfer mechanisms using Buongiorno's model in a MHD Carreau nanofluid flow past a permeable stretching surface. Rama Subba Reddy Gorla and Ali Chamkha [26] analyzed the natural convection via a non-isothermal vertical plate in a porous medium saturated by a nanofluid with thermophoresis and Brownian motion effects. Chamkha and Aly [27] discussed the magnetohydrodynamic free convective flow of a nanofluid over a vertical plate with heat generation / absorption effects.

Chemical reaction effects on steady/unsteady motion of fluids through porous medium along with behaviors of physical aspects discussed by many authors. A few of them are presented here. Chamkha et al. [28] presented the effects of chemical reaction on unsteady heat and mass transfer via a permeable stretching surface. They have shown the similarity solutions for the both suction/ injection cases. Hsiao [29] has been reported an article to study the radiative electrical MHD activation energy thermal extrusion manufacturing system efficiency with the help of Carreau-Nanofluid and parameters control method. Arrhenius activation energy in binary chemical reactions in the studies of non-Newtonian fluids is also found in literature with different physical effects. Irfan et al. [30] examined the three dimensional Carreau nanofluid along with the effects of nonlinear mixed convection 
and thermal radiation influenced by Arrhenius activation energy in chemical reaction. Irfan et al. [31] also discussed the dual solutions of a magnetohydrodynamic nonlinear radiative time dependent Carreau fluid along with the influence of activation energy in binary chemical reaction. Recently Alsaadi et al. [32] reported on Arrhenius activation energy and the effects of nonlinear radiative MHD Williamson fluid flow through a surface which is convectively heated.

In the present article, a novel modeling for a steady two-dimensional nonlinear convective flow, heat and mass transfer of Carreau nanofluid over an exponentially stretching permeable surface with viscous dissipation, applied magnetic field, Arrhenius activation energy involved in binary chemical reaction, convective boundary

\section{Mathematical formulation}

A steady two-dimensional magnetohydrodynamic boundary layer flow of an incompressible Carreau nanofluid over an exponentially stretching permeable surface with convective heat and mass conditions are considered. The effects of Brownian motion and thermophoresis are taken into consideration. Temperature, Concentration distributions and applied magnetic field are treated to be functions of exponential variation. Here $x$-axis is parallel to the surface and the $y$-axis is perpendicular to it (see Fig. 1).

The equations which are pertaining to the problem are:

$\frac{\partial u}{\partial x}+\frac{\partial v}{\partial y}=0$

$$
\begin{aligned}
u \frac{\partial u}{\partial x}+v \frac{\partial u}{\partial y}= & v \frac{\partial^{2} u}{\partial y^{2}}\left[1+\Gamma^{2}\left(\frac{\partial u}{\partial y}\right)^{2}\right]^{\frac{n-1}{2}}+v(n-1) \Gamma^{2} \frac{\partial^{2} u}{\partial y^{2}}\left(\frac{\partial u}{\partial y}\right)^{2}\left[1+\Gamma^{2}\left(\frac{\partial u}{\partial y}\right)^{2}\right]^{\frac{n-3}{2}} \\
& +g \beta_{T}\left(T-T_{\infty}\right)+g \beta_{C}\left(C-C_{\infty}\right)+g \beta_{1 T}\left(T-T_{\infty}\right)^{2}+g \beta_{1 C}\left(C-C_{\infty}\right)^{2}-\frac{\sigma B^{2}(x)}{\rho} u
\end{aligned}
$$

conditions is studied numerically and the outcomes are reported. Effects of Brownian motion and thermophoresis are taken into consideration. Authors strongly believe that no such modeling was attempted earlier. Governing equations of the present model are converted into a set of ordinary differential equations using similarity transformation and then solved by shooting technique based on R-K 4th order. The responses of fluid velocity, both temperature and concentration domains for different parameters of the problem are presented through plots. Also, the values of Skin friction, Nusselt number and Sherwood number are displayed in tables. Comparison of the present results with the published results is discussed.

$$
u \frac{\partial T}{\partial x}+v \frac{\partial T}{\partial y}=\frac{k}{\rho c_{p}} \frac{\partial^{2} T}{\partial y^{2}}+\frac{v}{c_{p}}\left(\frac{\partial u}{\partial y}\right)^{2}+\tau\left[D_{B} \frac{\partial C}{\partial y} \frac{\partial T}{\partial y}+\frac{D_{T}}{T_{\infty}}\left(\frac{\partial T}{\partial y}\right)^{2}\right]
$$

$$
u \frac{\partial C}{\partial x}+v \frac{\partial C}{\partial y}=D_{B} \frac{\partial^{2} C}{\partial y^{2}}+\frac{D_{T}}{T_{\infty}} \frac{\partial^{2} T}{\partial y^{2}}-k_{r}^{2}\left(C-C_{\infty}\right)\left(\frac{T}{T_{\infty}}\right)^{m} \exp \left(-\frac{E_{a}}{k^{*} T}\right)
$$

and accompanying conditions are

$$
\begin{array}{cc}
u=U_{w}(x)+N(x) v \frac{\partial u}{\partial y}, v=-V_{w}(x), T=T_{w}(x), C=C_{w}(x), \\
-k \frac{\partial T}{\partial y}=h_{1}\left(T_{0}-T\right),-D_{B} \frac{\partial C}{\partial y}=h_{2}\left(C_{0}-C\right) & \text { at } y=0 \\
u=0, T \rightarrow T_{\infty}, C \rightarrow C_{\infty} & \text { as } y \rightarrow \infty
\end{array}
$$

Fig. 1 Schematic diagram for flow pattern

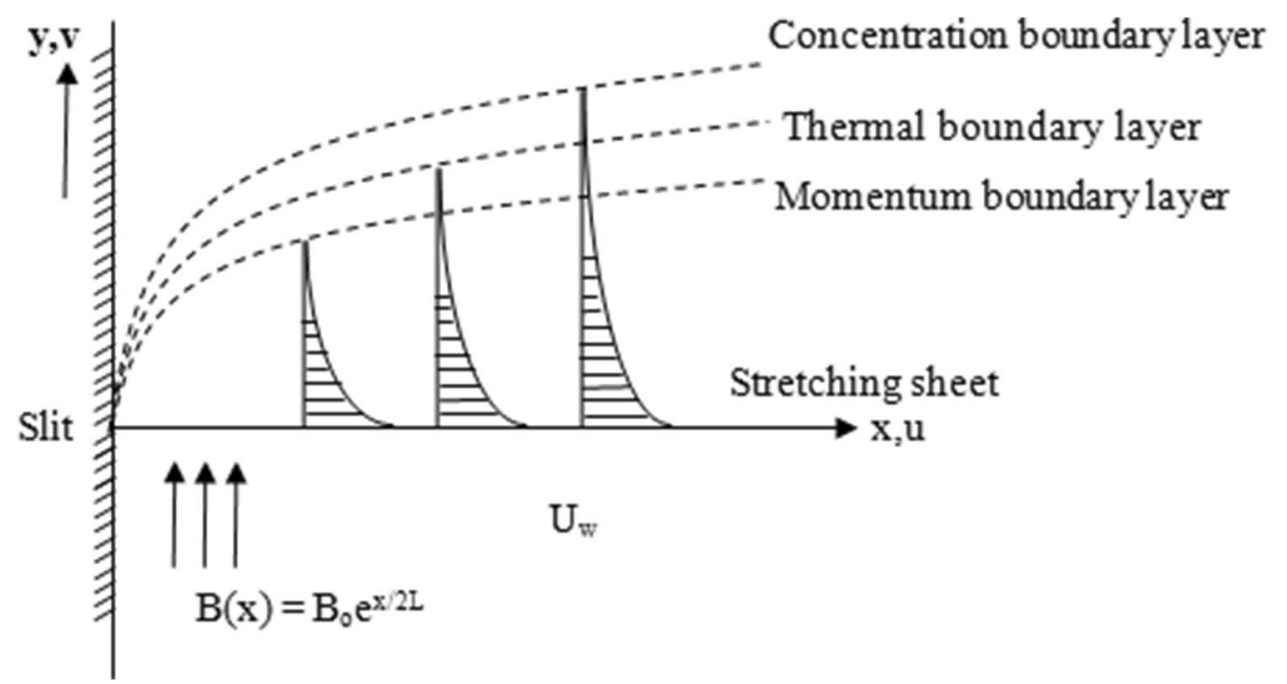


Here $h_{1}, h_{2}$ are taken to be convective heat transfer and mass transfer coefficients respectively, $k$ denotes thermal conductivity, $N(x)=N_{1} e^{-x / 2 L}$ is denoted as velocity slip factor and $N_{1}$ is the initial value of velocity slip factor. Here $V_{w}(x)=V_{0} e^{x / 2 L}$ is a velocity at the wall, where $V_{0}$ is the initial strength of suction. Here $V_{w}(x)>0$ indicates suction $V_{w}(x)<0$ indicates injection (see reference [7]).

The stretching velocity $U_{w}$, the temperature and concentration distributions at the wall, applied magnetic field are described below.

$$
\begin{aligned}
U_{w}(x)=U_{0} e^{x / L} ; T_{w}(x)= & T_{\infty}+\left(T_{0}-T_{\infty}\right) e^{(2 x / L)} ; C_{w}(x) \\
& =C_{\infty}+\left(C_{0}-C_{\infty}\right) e^{(2 x / L)}, B(x)=B_{0} e^{x / 2 L}
\end{aligned}
$$

The similarity variables are

$$
\begin{aligned}
\eta= & y \sqrt{\frac{U_{0}}{2 L v}} e^{x / 2 L}, \psi(x, y)=\sqrt{2 L U_{0} v} e^{x / 2 L} f(\eta) \\
& T(x, y)=T_{\infty}+\left(T_{0}-T_{\infty}\right) e^{(2 x / L)} \theta(\eta), C(x, y) \\
& =C_{\infty}+\left(C_{0}-C_{\infty}\right) e^{(2 x / L)} \phi(\eta)
\end{aligned}
$$

Through the stream function $\psi$, we defined $u=\frac{\partial \psi}{\partial y}$ and $v=-\frac{\partial \psi}{\partial x}$.

Using (6) in the Eqs. (2)-(4), we got

$$
\begin{aligned}
(1 & \left.+W e^{2} f^{\prime \prime 2}\right)^{\frac{n-3}{2}}\left(1+n W e^{2} f^{\prime \prime 2}\right) f^{\prime \prime \prime}+f f^{\prime \prime}-2 f^{\prime 2} \\
& +2 \lambda e^{2 X}\left[\theta\left(1+\gamma_{t} \theta e^{2 X}\right)+M^{*} \phi\left(1+\gamma_{c} \phi e^{2 X}\right)\right]-2 M f^{\prime}=0
\end{aligned}
$$

$\theta^{\prime \prime}+\operatorname{Pr}\left(f \theta^{\prime}-4 f^{\prime} \theta\right)+E c \cdot P r \cdot f^{\prime \prime 2}+\operatorname{Pr} \cdot e^{2 X}\left[N_{t} \theta^{2}+N_{b} \theta^{\prime} \phi^{\prime}\right]=0$

$$
\begin{aligned}
& \phi^{\prime \prime}+\operatorname{Sc}\left(f \phi^{\prime}-4 f^{\prime} \phi\right)+\left(\frac{N_{t}}{N_{b}}\right) \theta^{\prime \prime}-S c \Lambda e^{-X} \\
& \phi\left(1+\Lambda^{*} e^{2 X} \theta\right)^{m} \exp \left[\frac{-E}{1+\Lambda^{*} e^{2 X} \theta}\right]=0
\end{aligned}
$$

Also the reduced conditions are

$$
\begin{gathered}
f=S, f^{\prime}=1+P f^{\prime \prime}, \theta=\left(\frac{1}{B i_{T}}\right) \theta^{\prime}+e^{-2 X}, \\
\phi=\left(\frac{1}{B i_{C}}\right) \phi^{\prime}+e^{-2 X} \quad \text { at } \eta=0 \\
f^{\prime} \rightarrow 0, \theta \rightarrow 0, \phi \rightarrow 0 \text { as } \eta \rightarrow \infty
\end{gathered}
$$

The parameters used in this problem are listed below: $X=x / L$ is dimensionless coordinate for the plate; $P=N_{1} \sqrt{\frac{U_{0} v}{2 L}}$ is velocity slip parameter; $S=v_{0} \sqrt{\frac{2 L}{U_{0} v}}$ is the suction/injection parameter; $w e=\sqrt{\frac{\Gamma^{2} U_{0}^{3} e^{(3 \times / L)}}{2 L L}}$ is the local
Weissenberg number; $\lambda=\frac{G r_{T}}{R e^{2}}=\frac{g \beta_{T}\left(T_{W}-T_{\infty}\right) L^{3}}{\nu^{2}}$ is mixed convection parameter; ${ }_{M^{*}}=\frac{G r_{C}}{G r_{T}}$ is a ratio of the concentration to the thermal buoyancy forces; $\gamma_{t}=\frac{\beta_{1 T}\left(T_{w}-T_{\infty}\right)}{\beta_{T}}$ is the nonlinear convection parameter caused by temperature; $\gamma_{c}=\frac{\beta_{1 C}\left(C_{w}-C_{\infty}\right)}{\beta_{c}}$ is the nonlinear convection parameter caused by concentration; $N_{b}=\frac{\tau D_{B}\left(C_{w}-C_{\infty}\right)}{v}$ is a parameter due to Brownian motion; $N_{t}=\frac{\tau D_{T}\left(T_{w}-T_{\infty}\right)}{\nu T_{\infty}}$ is a parameter due to thermophoresis; $\operatorname{Pr}=v / \alpha$ is Prandtl number; $E C=\frac{U_{0}^{2}}{c_{p}\left(T_{0}-T_{\infty}\right)}$ is Eckert number; $S C=\frac{v}{D_{B}}$ is the Schmidt number; $\Lambda=\frac{2 L K_{r}^{2}}{U_{0}}$ is chemical reaction rate; $\Lambda^{*}=\frac{T_{0}-T_{\infty}}{T_{\infty}}$ is temperature ratio parameter; $E=\frac{E_{a}}{k^{*} T_{\infty}}$ is the non-dimensional Arrhenius chemical energy; $M=\frac{H a^{2}}{R e}=\frac{\sigma B_{0}^{2} L}{\rho U_{0}}$ is magnetic field parameter; $B i_{T}=\frac{\mathrm{h}_{1} \mathrm{y}}{\eta \mathrm{k}}, B i_{C}=\frac{\mathrm{h}_{2} \mathrm{y}}{\eta \mathrm{k}}$ are the Biot numbers. Here $H a=\sqrt{\sigma B_{0}^{2} L^{2} / \rho v}$ is the Hartmann number; $R e=\frac{u_{0} L}{v}$ is Reynolds number. Also ' $n$ ' is the powerlaw index and ' $m$ ' is the fitted rate constant.

The physical parameters of engineering interest, friction factor $C_{f x}$ local Nusselt and Sherwood numbers i.e., $N u_{x}$ and $S h_{x}$ are specified as

$C_{f x}=\frac{\tau_{w}}{\rho u_{w}^{2}}, N u_{x}=\frac{x q_{w}}{k\left(T_{w}-T_{\infty}\right)}, S h_{x}=\frac{x j_{w}}{D_{B}\left(C_{w}-C_{\infty}\right)}$

Here $\tau_{w} q_{w}$ and $j_{w}$ stands for wall shear stress, heat and mass flux correspondingly, which are specified below.

$$
\begin{gathered}
\tau_{w}=\left.\mu \frac{\partial u}{\partial y}\left[1+\Gamma^{2}\left(\frac{\partial u}{\partial y}\right)^{2}\right]^{n-1 / 2}\right|_{y=0}, \\
q_{w}=-\left.k\left(\frac{\partial T}{\partial y}\right)\right|_{y=0}, j_{w}=-\left.D_{B}\left(\frac{\partial C}{\partial y}\right)\right|_{y=0}
\end{gathered}
$$

In non-dimensional form they prescribed as

$$
\begin{aligned}
\sqrt{R e_{x}} C_{f x} & =\sqrt{2} f^{\prime \prime}(0)\left[1+W e\left(f^{\prime \prime}(0)\right)^{2}\right]^{(n-1) / 2}, \\
& \frac{N u_{x}}{\sqrt{X / 2} \sqrt{R e_{x}}}=-\theta^{\prime}(0), \frac{S h_{x}}{\sqrt{X / 2} \sqrt{R e_{x}}}=-\phi^{\prime}(0)
\end{aligned}
$$

Local Reynold's number, $\operatorname{Re}_{x}=\frac{x U_{w}(x)}{v}$. 


\section{Numerical approach}

Numerical Solutions for converted system of Eqs. (7)-(9) along with conditions (10) are achieved through shooting technique based on $\mathrm{R}-\mathrm{K} 4$ th order procedure. For this process, equations are altered into a set of first order ordinary differential equations using the meaningful replacements as mentioned below:

$f_{1}=f$,

$f_{2}=\frac{d f_{1}}{d \eta}$ or $\frac{d f}{d \eta}$

$f_{3}=\frac{d f_{2}}{d \eta}$ or $\frac{d^{2} f}{d \eta^{2}}$

$f_{4}=\theta(\eta)$,

$f_{5}=\frac{d f_{4}}{d \eta}$ or $\frac{d \theta}{d \eta}$

$f_{6}=\phi(\eta)$,

$f_{7}=\frac{d f_{6}}{d \eta}$ or $\frac{d \phi}{d \eta}$.

Making use of above, we write
Table 1 Numerical values of skin friction for various values of $W e, M, \lambda, n$ and $S$

\begin{tabular}{clllll}
\hline We & $M$ & $\lambda$ & $n$ & \multicolumn{2}{l}{$C_{f x}$} \\
\cline { 4 - 6 } & & & & $S=0.5$ & $S=-0.5$ \\
\hline 0.2 & 1.0 & 1.0 & 4.0 & -2.553236 & -1.764808 \\
0.5 & - & - & - & -1.662398 & -1.328209 \\
0.7 & - & - & - & -1.391724 & -1.156398 \\
0.5 & 1.0 & 1.0 & 4.0 & -1.662867 & -1.328476 \\
- & 2.0 & - & - & -1.891976 & -1.641021 \\
- & 3.0 & - & - & -2.057223 & -1.851347 \\
0.5 & 1.0 & 1.0 & 4.0 & -1.662867 & -1.328476 \\
- & - & 1.5 & - & -1.533758 & -1.118731 \\
- & - & 2.0 & - & -1.386599 & -0.847425 \\
0.5 & 1.0 & 1.0 & 2.0 & -2.909279 & -1.817405 \\
- & - & - & 3.0 & -1.999158 & -1.498077 \\
- & - & - & 4.0 & -1.662398 & -1.328209 \\
\hline
\end{tabular}

For the responses of the system, we employed a shooting technique depending on RK $4^{\text {th }}$ order method. For one set of values of the parameters, values of $k_{1}, k_{2}$ and $k_{3}$ are assumed and the system of first order ordi-

$$
\begin{aligned}
& \frac{d f_{1}}{d \eta}=f_{2,} \\
& \frac{d f_{2}}{d \eta}=f_{3,} \\
& \frac{d f_{3}}{d \eta}=\frac{2\left(f^{\prime}\right)^{2}-f f^{\prime \prime}-2 \lambda e^{2 X}\left[\theta\left(1+\gamma_{t} e^{2 X} \theta\right)+M^{*} \phi\left(1+\gamma_{c} e^{2 X} \phi\right)\right]+2 M f^{\prime}}{\left(1+W e^{2}\left(f^{\prime \prime}\right)^{2}\right)^{(n-3) / 2}\left(1+n W e^{2}\left(f^{\prime \prime}\right)^{2}\right)}, \\
& \frac{d f_{4}}{d \eta}=f_{5}, \\
& \frac{d f_{5}}{d \eta}=\operatorname{Pr}\left(4 f^{\prime} \theta-f \theta^{\prime}\right)-E c \cdot \operatorname{Pr} \cdot\left(f^{\prime \prime}\right)^{2}-\operatorname{Pr} e^{2 X}\left[N_{t} \theta^{\prime 2}+N_{b} \theta^{\prime} \phi^{\prime}\right], \\
& \frac{d f_{6}}{d \eta}=f_{7}, \\
& \frac{d f_{7}}{d \eta}=\operatorname{Sc}\left(4 f^{\prime} \phi-f \phi^{\prime}\right)-\left(\frac{N_{t}}{N_{b}}\right) \theta^{\prime \prime}+\mathrm{Sc} \cdot \Lambda \cdot e^{-X} \phi\left(1+\Lambda^{*} e^{2 X} \theta\right)^{m} \exp \left[\frac{-E}{1+\Lambda^{*} e^{2 X} \theta}\right],
\end{aligned}
$$

The corresponding initial conditions will be

$$
\begin{aligned}
& f_{1}(0)=S, f_{2}(0)=1+P f_{3}(0), f_{3}(0)=k_{1}, f_{4}(0)=k_{2}, \\
& f_{5}(0)=B i_{T}\left(k_{2}-e^{-2 X}\right) f_{6}(0)=k_{3} f_{7}(0)=B i_{C}\left(k_{3}-e^{-2 X}\right)
\end{aligned}
$$

nary differential equations is solved as an initial value problem from $\eta=0$ to $\eta=\eta_{\infty}\left(\eta_{\infty}\right.$ is a large value of $\eta$ and $\left.\eta_{\infty}: 4-12\right)$ using RK method. The values of $f^{\prime}, \theta$ and $\phi$ at $\eta_{\infty}$ are compared with their expected values and 
accordingly $k_{1}, k_{2}$ and $k_{3}$ are modified and the system of equations again solved as an initial value problem. The procedure is repeated a number of times till the values of $f^{\prime}, \theta$ and $\phi$ at $\eta_{\infty}$ are very close to the expected values. By the above mentioned procedure we can get values of $k_{1}, k_{2}$ and $k_{3}$ for one set of values of the parameters of the study. Similarly we determined the values of $k_{1}, k_{2}$ and $k_{3}$ for all possible values of the parameters of the study. Using the initial conditions, the equations are solved to get the velocity, temperature and concentration distributions and also the quantities like skin friction, heat and mass transfer rates. During this process, the step size and convergence criteria are maintained as 0.001 and $10^{-6}$ in all cases.
Table 2 Numerical values of Nusselt number for various values of $M, \lambda, B i_{T}, E c, N_{t}, N_{b}$ and $S$
Table 3 Numerical values of Sherwood number for various values of $S c, E, B i_{C}, N_{t}, N_{b}$ and $S$

\begin{tabular}{llllllll}
\hline$M$ & $\lambda$ & $B i_{T}$ & $E C$ & $N_{t}$ & $N_{b}$ & \multicolumn{2}{l}{$N u_{x}$} \\
\cline { 5 - 7 } & & & & & & $S=0.5$ & $S=-0.5$ \\
\hline 1.0 & 1.0 & 2.0 & 0.002 & 0.1 & 0.1 & 0.332810 & 0.297811 \\
3.0 & - & - & - & - & - & 0.327676 & 0.289923 \\
1.0 & - & - & - & - & - & 0.320621 & 0.283866 \\
- & 1.0 & 2.0 & 0.002 & 0.1 & 0.1 & 0.332810 & 0.297811 \\
- & 1.5 & - & - & - & - & 0.336314 & 0.301954 \\
1.0 & 2.0 & - & - & - & - & 0.341251 & 0.307400 \\
- & 1.0 & 0.3 & 0.002 & 0.1 & 0.1 & 0.092932 & 0.089648 \\
- & - & 0.6 & - & - & - & 0.161132 & 0.151918 \\
1.0 & - & 0.9 & - & - & - & 0.213482 & 0.197984 \\
- & 1.0 & 2.0 & 0.02 & 0.1 & 0.1 & 0.327230 & 0.294277 \\
- & - & - & 0.03 & - & - & 0.324192 & 0.292350 \\
1.0 & - & - & 0.04 & - & - & 0.321197 & 0.290450 \\
- & 1.0 & 2.0 & 0.002 & 0.1 & 0.1 & 0.288544 & 0.261534 \\
- & - & - & - & 0.2 & - & 0.285902 & 0.259746 \\
1.0 & - & - & - & 0.3 & - & 0.282513 & 0.257759 \\
- & 1.0 & 2.0 & 0.002 & 0.1 & 0.1 & 0.225668 & 0.208063 \\
- & - & - & - & - & 0.2 & 0.223436 & 0.205943 \\
\hline & - & - & - & - & 0.3 & 0.220393 & 0.203077 \\
\hline
\end{tabular}

\begin{tabular}{lllllll}
\hline$S c$ & $E$ & $B i_{C}$ & $N_{t}$ & $N_{b}$ & \multicolumn{3}{l}{$S h_{x}$} & \\
\cline { 3 - 6 } & & & & & $S=0.5$ & $S=-0.5$ \\
\hline 0.2 & 1.0 & 2.0 & 0.1 & 0.1 & 0.078805 & 0.100919 \\
0.3 & - & - & - & - & 0.108272 & 0.125119 \\
0.4 & - & - & - & - & 0.133868 & 0.146053 \\
0.2 & 0.2 & 2.0 & 0.1 & 0.1 & 0.034257 & 0.062879 \\
- & 0.4 & - & - & - & 0.025694 & 0.055891 \\
- & 0.6 & - & - & - & 0.017647 & 0.049443 \\
0.2 & 1.0 & 0.3 & 0.1 & 0.1 & 0.042391 & 0.045222 \\
- & - & 0.6 & - & - & 0.064985 & 0.068882 \\
- & - & 0.9 & - & - & 0.078973 & 0.083399 \\
0.2 & 1.0 & 2.0 & 0.05 & 0.1 & 0.127499 & 0.133026 \\
- & - & - & 0.07 & - & 0.099310 & 0.109764 \\
- & - & - & 0.09 & - & 0.071007 & 0.086473 \\
0.2 & 1.0 & 2.0 & 0.1 & 0.1 & 0.171196 & 0.169345 \\
- & - & - & - & 0.2 & 0.182362 & 0.178659 \\
- & - & - & - & 0.3 & 0.189058 & 0.184275 \\
\hline
\end{tabular}


Table 4 Variations in $f^{\prime \prime}(0)$ for different values of $M$ when $n=W e=S=\lambda=0$

\begin{tabular}{lll}
\hline$M$ & $f^{\prime \prime}(0)$ & $f^{\prime \prime}(0)$ \\
& Ref. [24] & Present work \\
\hline 0.0 & -1.281828 & -1.281832 \\
0.2 & -1.313294 & -1.313286 \\
0.4 & -1.403028 & -1.403045 \\
\hline
\end{tabular}

Table 5 Variations in $-\theta^{\prime}(0)$ for different values of $\operatorname{Pr}$ when $W e=S=M=N_{t}=N_{b}=E C=X=0$ and $n=1$

\begin{tabular}{lll}
\hline $\operatorname{Pr}$ & $\begin{array}{l}-\theta^{\prime}(0) \\
\text { Ref. [25] }\end{array}$ & $\begin{array}{l}-\theta^{\prime}(0) \\
\text { Present work }\end{array}$ \\
\hline 0.72 & 1.088915 & 1.088920 \\
1.00 & 1.333333 & 1.333328 \\
3.00 & 2.509698 & 2.509689 \\
10.00 & 4.796853 & 4.796845 \\
\hline
\end{tabular}



Fig. 2 Effect of Weissenberg number on velocity

\section{Results and discussion}

After employing shooting method (based on RK 4th order) on the converted system of Eqs. (7)-(9) including the conditions prescribed in (10), the responses of fluid velocity, temperature and concentration domains for distinct important physical parameters in both suction $(S>0)$ and injection $(S<0)$ cases are deliberated through plots. Contour plots and streamline plots are also displayed. The friction coefficient, Nusselt number and Sherwood number computational values are presented in Tables 1,2 and 3 for suction and injection regions. Validity of the present study (Tables 4 and 5 ) is discussed at the end of the discussion

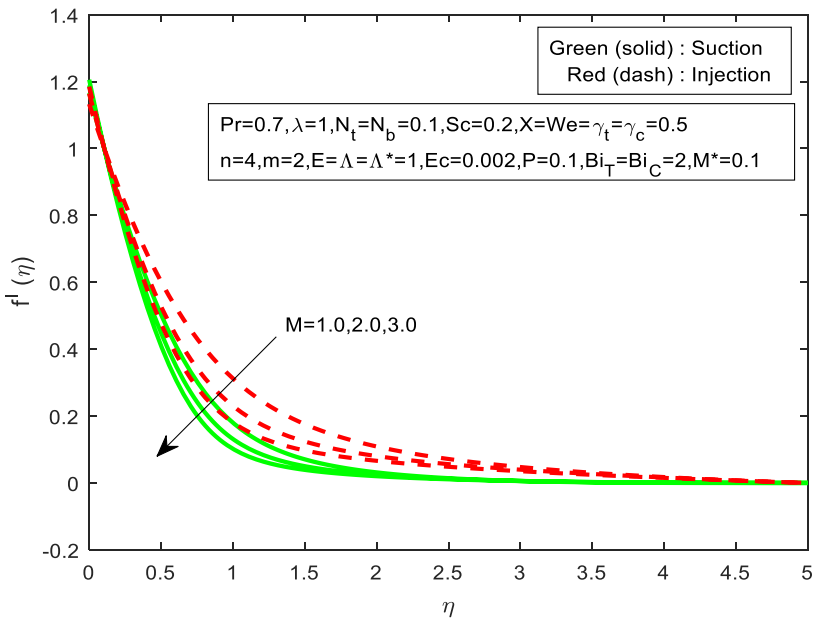

Fig. 3 Effect of magnetic parameter on velocity

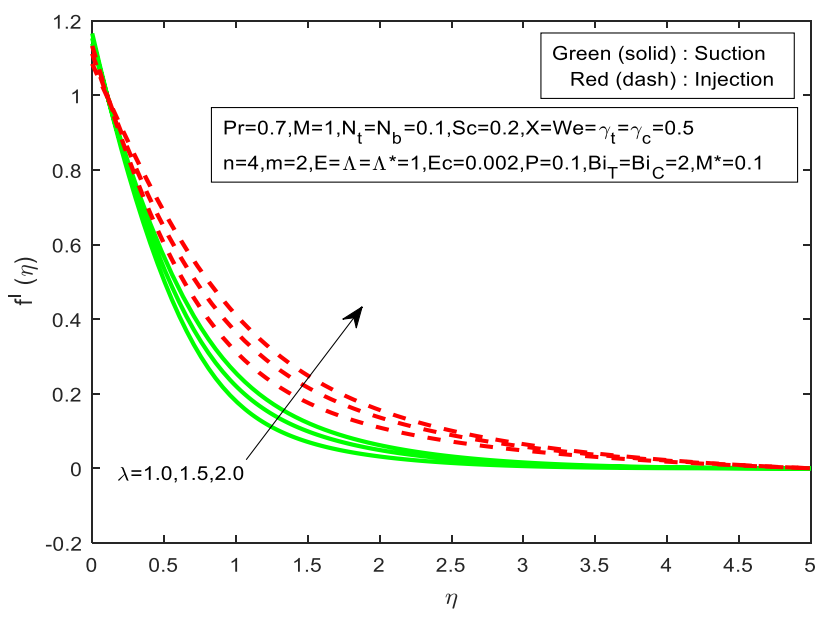

Fig. 4 Effect of mixed convection on velocity

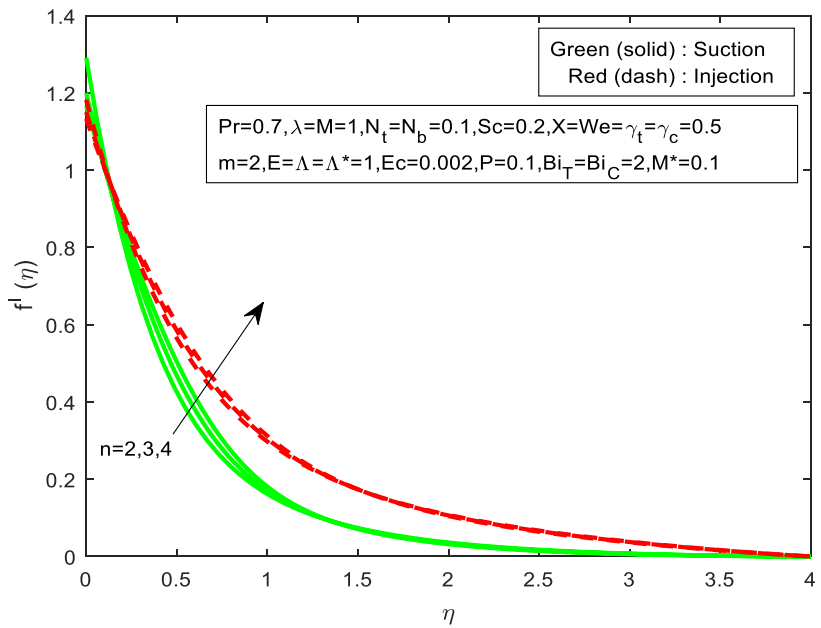

Fig. 5 Effect of power-law index on velocity 



a
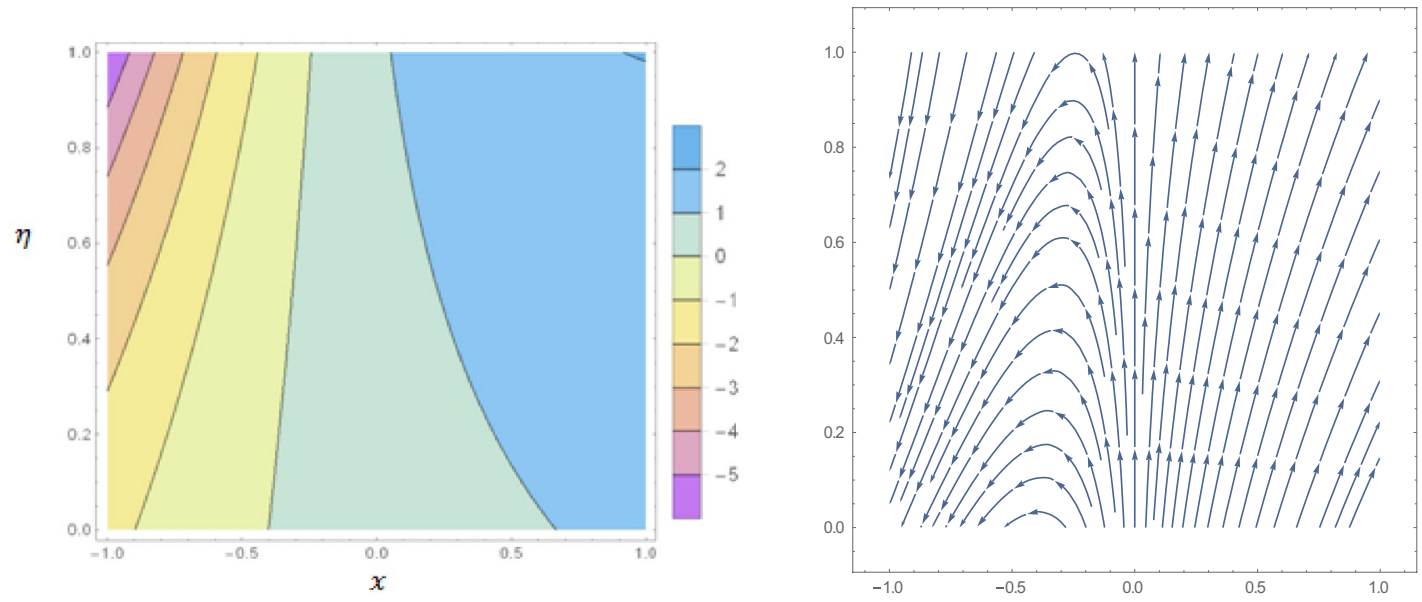

b
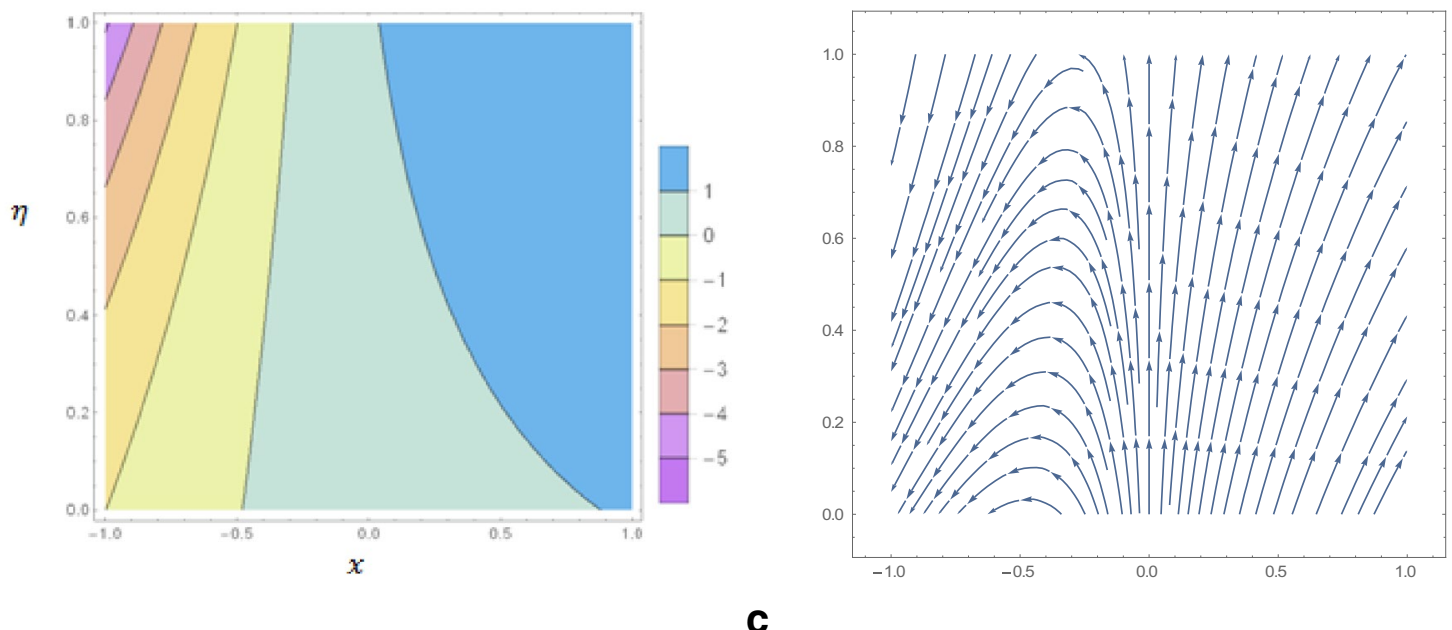
४Fig. 6 Contour plot and stream lines (for values of $f$ ). $\mathbf{a}[\operatorname{Pr}=0.7$,$] . \mathbf{b}$ $\lambda=M=E=\Lambda=\Lambda^{*}=1, N_{t}=N_{b}=0.1, S=0.5, S c=0.2, X=1$. c $W e=\gamma_{t}=\gamma_{c}=0.5, n=4, m=2, \mathrm{Ec}=0.002, \mathrm{P}=0.1, \mathrm{Bi}_{\mathrm{T}}=\mathrm{Bi}_{\mathrm{C}}$ $\left.=2, \mathrm{M}^{*}=0.1\right]$

of the results. The results are calculated for a particular set of values of the parameters which are mentioned in figures. If $n>1$, it indicates the shear thickening case and if $0<n<1$, it indicates the shear thinning case. Results are presented for shear thickening case $(n=4)$ in the presence of suction/injection.

\subsection{Velocity profile}

The consequences in fluid velocity with the parameters We, $M, \lambda$ and $n$ for both suction and injection regions are displayed in Figs. 2, 3, 4 and 5. Figure 2 reveals the influence of Weissenberg number (We) on velocity. Increased values of We heightens the profiles of velocity in shear thickening fluids. As per the definition of Weissenberg number it is the ratio of the relaxation time of the fluid and a specific process time and in the case of shear thickening fluids $(n>1)$, due to thickness of the fluid is diminished, so that the fluid velocity is slightly enhanced. The magnetic field impact on fluid velocity is shown in Fig. 3. It reveals that the larger values of magnetic parameter depreciate the velocity. Also the magnetic parameter depresses the momentum boundary layer thickness. Since the magnetic parameter is the ratio of electromagnetic force to the viscous force and so the increased magnetic effect leads to occurrence of Lorentz forces, as a result motion of the fluid gets obstructed and hence velocity is decreased. Variations in velocity of the fluid due to mixed convection parameter shown in Fig. 4. The enhancement in mixed convection leads to the intensification of fluid velocity. This happens due to larger values of mixed convection contributes enrichment of buoyancy forces, as a result velocity increased. Figure 5 is plotted to show the effect of $n$ (power law index) on velocity of the fluid. It was noticed that, the velocity drops almost exponentially with fixed values of $n$. But varying ' $n$ ', the velocity profiles are seen to be heightened with the same rate. Since the non-Newtonian aspects decreases with a rise in $n$ and as a result the velocity is enhanced. Contour plots and Streamlines are displayed in Fig. $6 a-c$ to observe the flow pattern.

\subsection{Temperature profile}

Temperature variations corresponding to the parameters $M, \lambda, E C, B i_{T}, N_{t}$ and $N_{b}$ are presented through the Figs. 7, 8, 9, 11 and 12. Mixed convection parameter versus temperature profile is shown in Fig. 7. Enhanced mixed convection causes decrement in temperature. When we increase the mixed convection parameter, the difference between temperature at the wall and free stream temperature also intensified. So temperature decreases. Figure 8 reveals the change in temperature due to magnetic effect. In this case also thermal boundary layer thickness is intensified with an enhancement in magnetic parameter. So that increment in $M$ causes the improvement in temperature profiles. Eckert number $(E C)$ versus temperature of the fluid is exhibited in Fig. 9. As ' $E C^{\prime}$ generates heat inside the flow region, fluid temperature gets elevated. In Fig. 10, the variations in temperature due to thermal Biot number $\left(B i_{T}\right)$ are shown. In physical point of view, an upgrade in $B i_{T}$, makes more grounded convection which prompts the elevated temperature. So that bigger values of $B i_{T}$ causes an increment in thermal boundary layer as result, the profiles of temperature are heightened. The impact of thermophoresis parameter $\left(N_{t}\right)$ on the dimensionless temperature in shear thickening case is displayed in Fig. 11. The enhancing trend in temperature is noticed with specified values of $N_{t}$. As, thermophoretic force is affected by the temperature gradient, the heated particles dragged away from hot to cold surface which causes raise in temperature field. In this case, vast amount of nanoparticles is transmuted from the intense surface which intensifies the temperature of Carreau fluid. The variations of temperature in response to a change in Brownian motion parameter $\left(N_{b}\right)$ is displayed in Fig. 12. Temperature enhances by uplifting the Brownian motion parameter. Moreover, thermal boundary layer thickness is a rising function of the Brownian motion parameter in shear thickening $(n>1)$ fluid. As per the definition of Brownian motion, increment in $N_{b}$, causes the larger kinetic energy of the nanoparticles and as result nanofluid temperature is rises.

\subsection{Concentration profile}

The changes in concentration profile with the parameters $S c, E, B i_{C}, N_{t}$ and $N_{b}$ are exhibited in Figs. $13,14,15,16$ and 17. Figure 13 displays the concentration field for the changing values of Schmidt number (Sc). From this figure, we see that the concentration of the fluid is decreased with bigger values of $S c$. The rate of decrease in concentration is larger for high values of Sc. Since the larger values of Schmidt number are equivalent to smaller mass diffusivity. So the reducing trend in concentration profiles is observed. Figure 14 is drafted to scrutinize the consequence of shear thickening liquids for activation energy parameter $(E)$ on concentration field. Enhancing values of ' $E$ ' causes a decline in the field of concentration. As a physical aspect, advanced values of ' $E$ ' falling-off the modified 


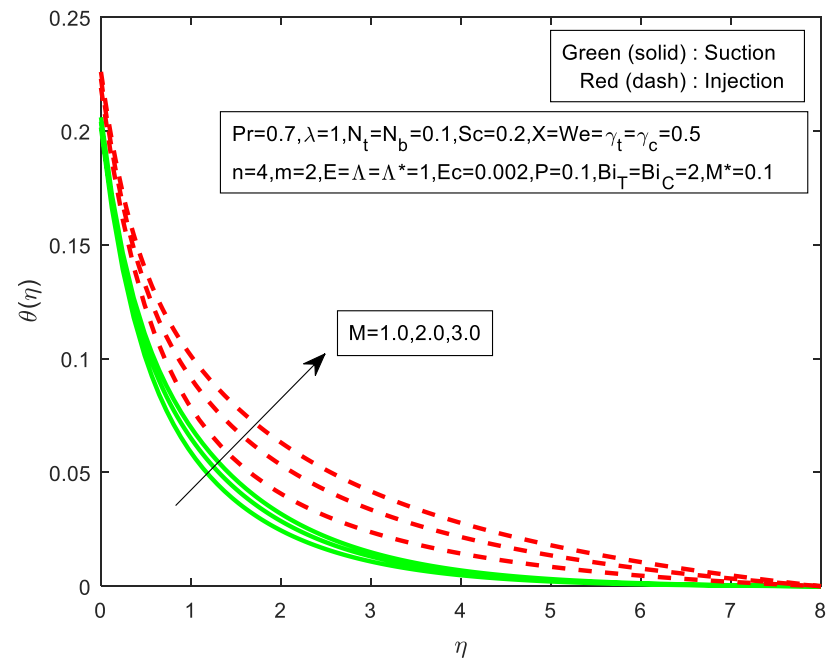

Fig. 7 Effect of magnetic parameter on temperature

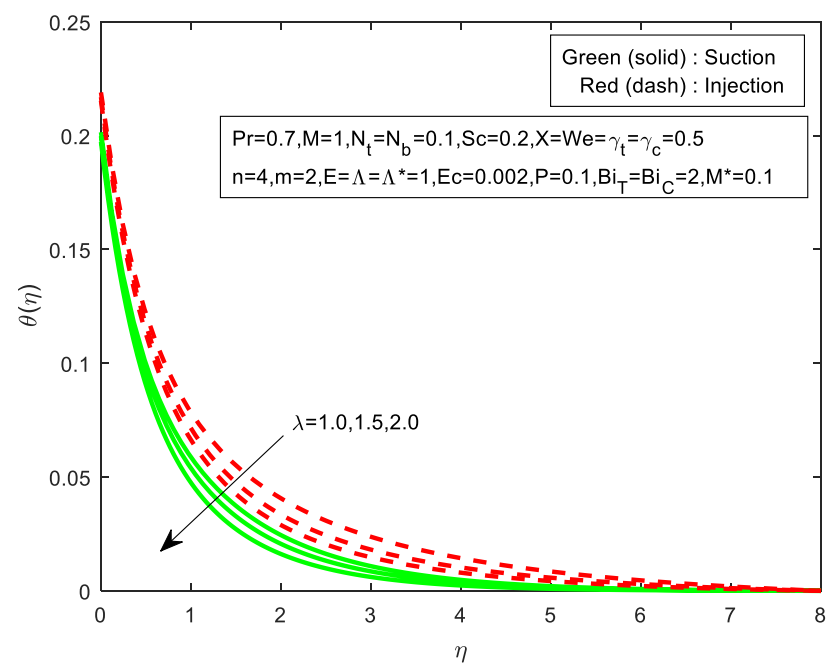

Fig. 8 Effect of mixed convection on temperature

Arrhenius function which stimulates the generative chemical reaction quantity. So that, the concentration of Carreau liquid is augments. The behavior of concentration profiles with mass transfer Biot number $\left(B i_{C}\right)$ is displayed in Fig. 15 . In both suction and injection regions, we noticed that the rising values of this Biot number, intensifies the concentration of fluid. As per the definition of Biot number, through convection the transferred mass will be distributed over the surface. So that nanofluid concentration profiles are intensified. Figures 16 and 17 are portrayed to know the impacts of both the parameters $N_{t}$ and $N_{b}$ on concentration field. For both the situations $(S>1$ and $S<1)$, the concentration of Carreau nanofluid far away from the wall boost up for growing values of $N_{t}$ (Fig. 16). The variation of concentration domain due to $N_{b}$ is visualized in Fig. 17. We

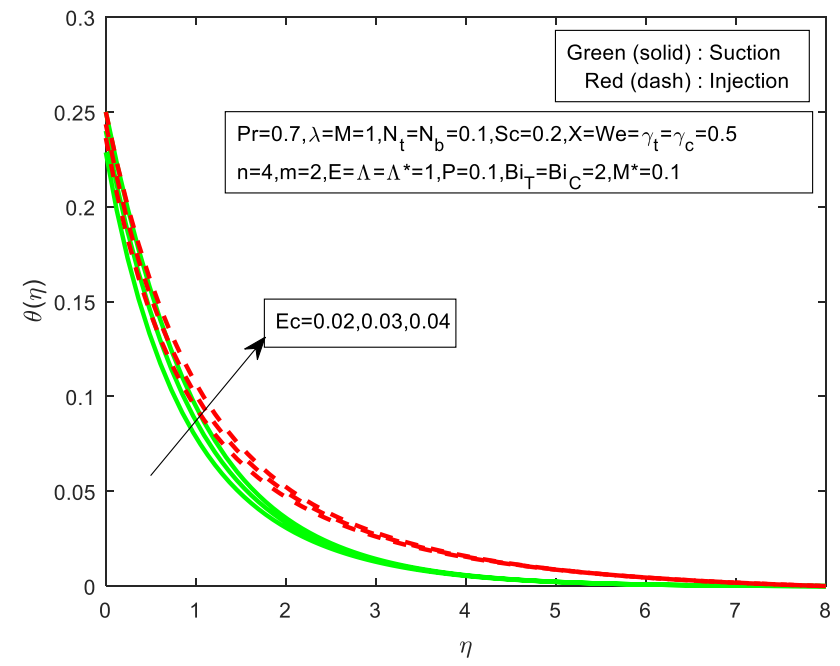

Fig. 9 Effect of Eckert number on temperature

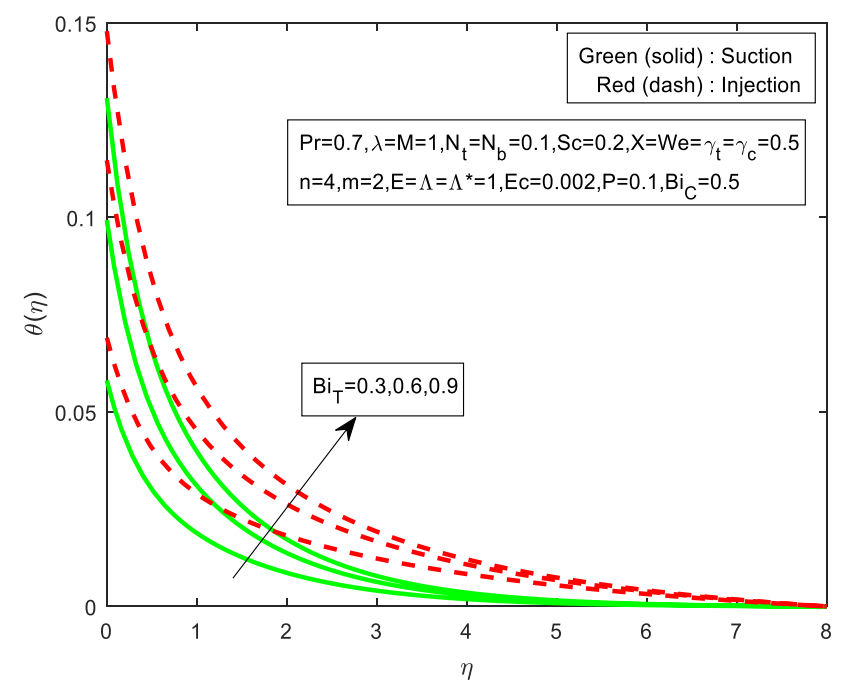

Fig. 10 Effect of thermal Biot number on temperature

observed from this figure that far away from the plate, concentration is diminished with a rising values of $N_{b}$ in both suction and injection regions. Physically, augmentation in $N_{b}$, the collision of macroscopic particles of a liquid intensifies due to the instance that $N_{b}$ reduces the concentration field far away from the wall.

Tables 1, 2 and 3 shows the computational values for friction coefficient, Nusselt number and Sherwood number for different values of parameters in both suction and injection regions. Growth in skin friction coefficient is noticed due to increment in local Weissenberg number. High magnetic effect decreases the friction coefficient and heat transfer rate. Enhanced mixed convection elevates the friction coefficient and transfer of heat. Skin friction is also intensified by high power-law index values. Increased 


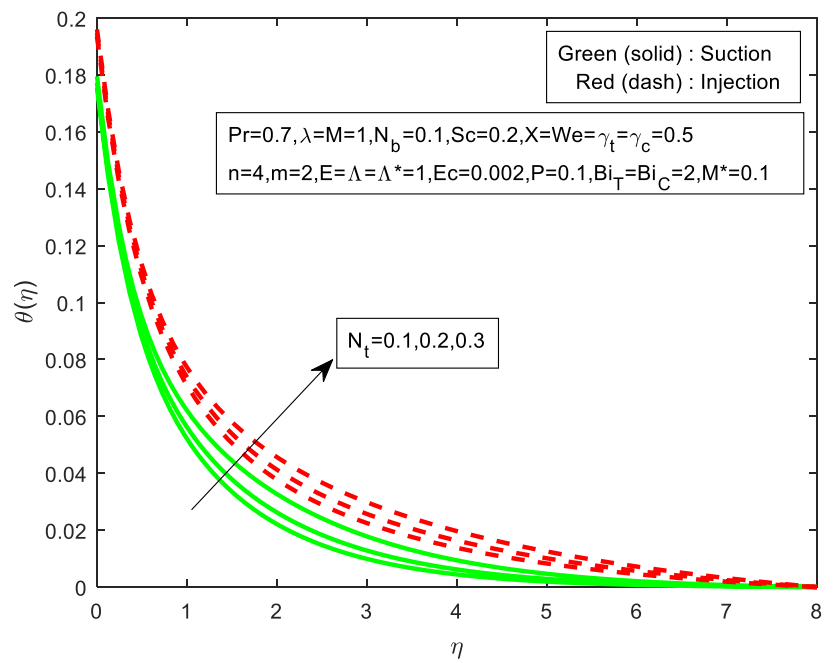

Fig. 11 Effect of thermophoresis on temperature

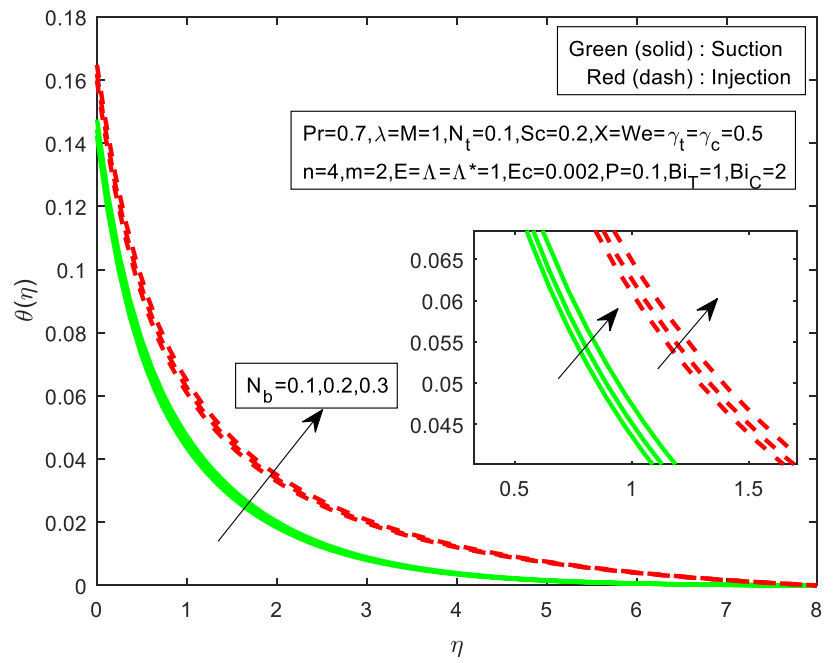

Fig. 12 Effect of Brownian motion on temperature

Brownian motion and thermophoresis elevates the heat transfer rate. Also Nusselt number is lowered by an increment in Eckert number but it is elevated by enhanced mixed convection and thermal Biot number. Sherwood number is lowered by a rise in thermophoresis effect and enhanced by improved Brownian motion. Larger values of Schmidt number and concentration Biot number results the growth in Sherwood number and it seen to be diminished by bigger values of activation energy parameter.

\subsection{Validity of the result}

Comparison of the numerical computations in this study with the published results in references $[24,25]$ is

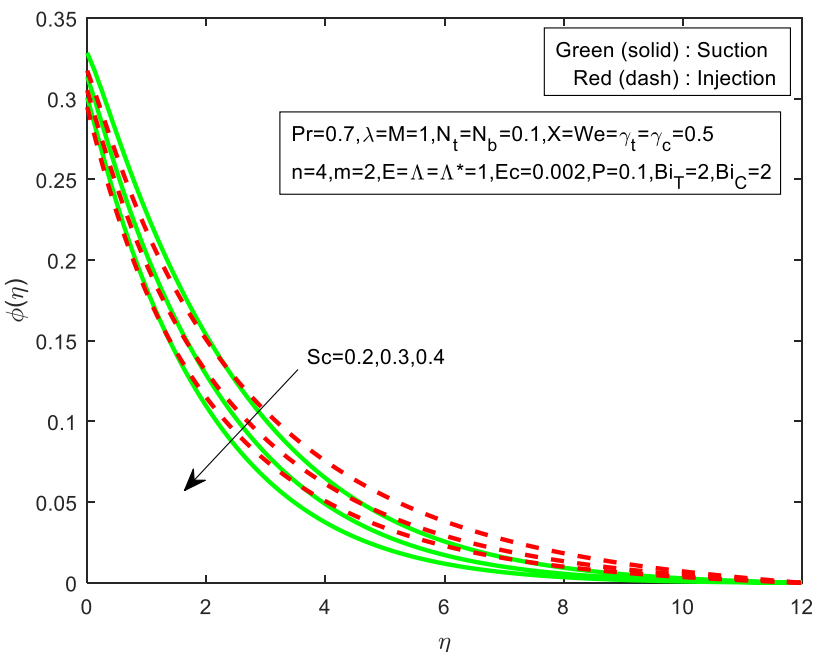

Fig. 13 Effect of schmidt number on concentration

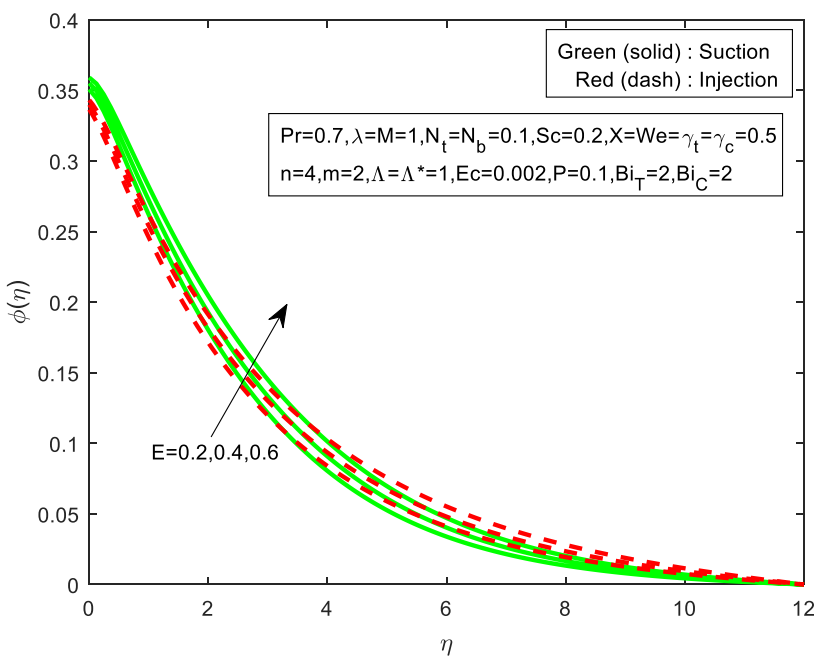

Fig. 14 Effect of activation energy on concentration

displayed through the tables as presented below. Table 4 describes the comparison in skin friction coefficient for varying values of the magnetic parameter ' $M$ ' and the values of other parameters are taken to be zero. The comparison in Nusselt number for various values of Prandtl number' $\operatorname{Pr}^{\prime}$ for $(n=1)$ and in the absence of other parameters is displayed in Table 5. These Computations are in good agreement with published results.

\section{Conclusions}

Numerical investigation on chemically reacting twodimensional steady incompressible and electrically conducting Carreau nanofluid through an exponentially 


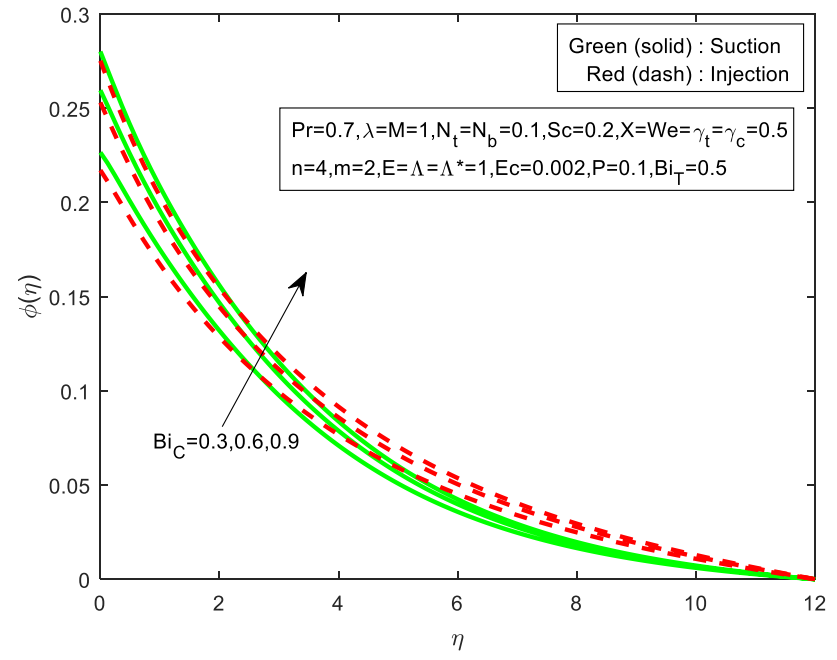

Fig. 15 Effect of mass transfer Biot number on concentration

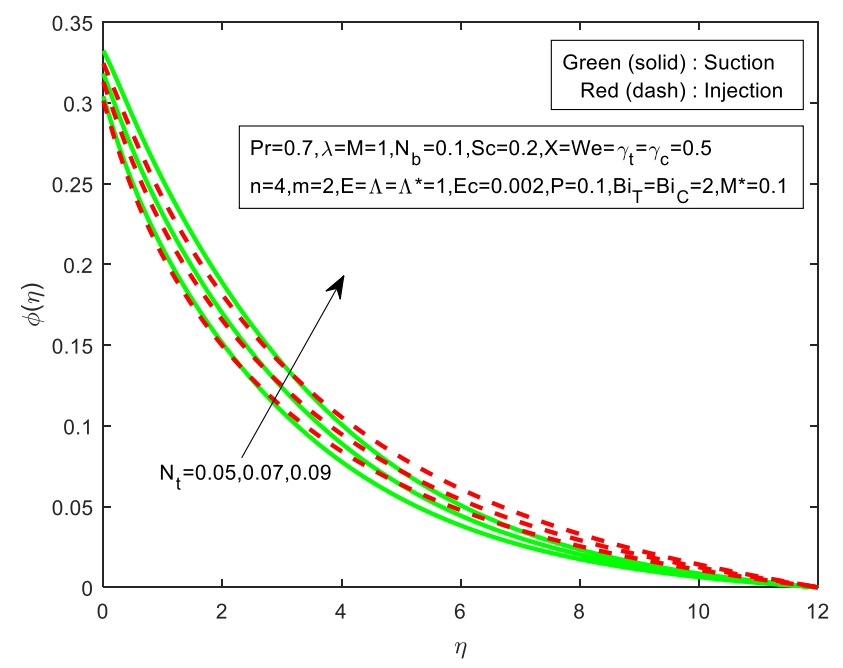

Fig. 16 Effect of thermophoresis on concentration

stretching permeable surface under chemical reaction with activation energy, viscous dissipation, slip velocity, nonlinear mixed convection has been carried out. Shooting method based on RK-method is used to solve the reduced system of equations with corresponding boundary conditions. Emphasis is on theoretical analysis only. This type of modeling is not found in literature. The model may be suitable for different fluids also. In general, if $n>1$ it is shear thickening case and if $0<n<1$, it is shear thinning case. Solutions are obtained for both suction and injection regions for the case $(n=4)$ for distinct values of

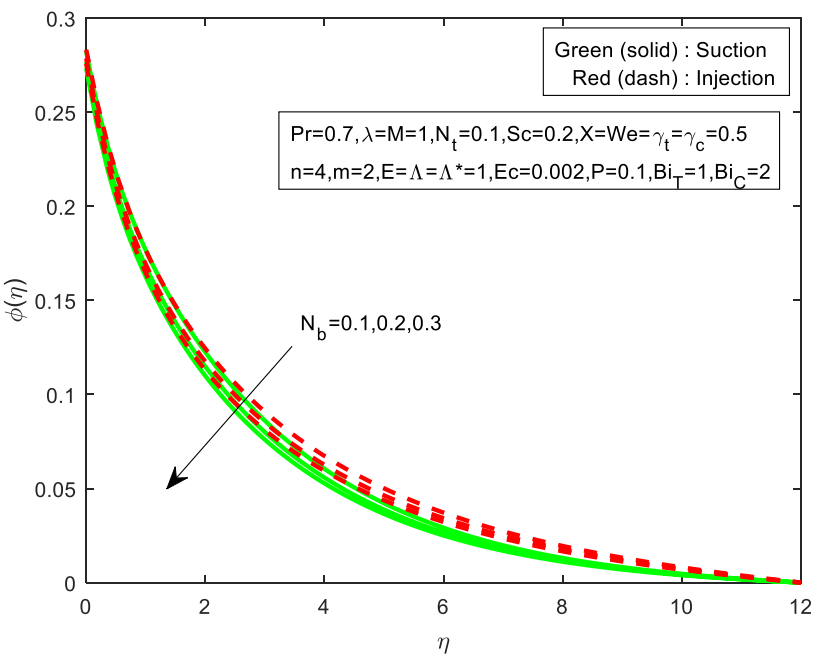

Fig. 17 Effect of Brownian motion on concentration

other parameters. For shear thinning case, solutions do not exist.

From this analysis, it can be concluded that the fluid velocity is an increasing function of local Weissenberg number. Momentum boundary layer thickness is depressed by magnetic field impact. Increasing trend in Carreau fluid temperature is noticed due to larger values of thermophoresis and Brownian motion effects. Concentration field is a decreasing function of Brownian motion but an increasing function of thermophoresis. Activation energy augments the concentration profile but Schmidt number lowers the same. Nusselt number is elevated by Brownian motion and thermophoresis effects. Heat transfer rate is lowered by an increment in Eckert number but it is elevated by enhanced mixed convection and thermal Biot number. Sherwood number is lowered by a rise in thermophoresis effect and enhanced by improved Brownian motion effect.

Acknowledgements The corresponding author conveys his special thanks to Editors and reviewers for their valuable suggestions to improve the quality of the paper.

\section{Compliance with ethical standards}

Conflict of interest On behalf of all authors, the corresponding author states that there is no "conflict of interest".

Open Access This article is licensed under a Creative Commons Attribution 4.0 International License, which permits use, sharing, adaptation, distribution and reproduction in any medium or format, as long as you give appropriate credit to the original author(s) and the source, provide a link to the Creative Commons licence, and indicate if changes were made. The images or other third party material in this article are included in the article's Creative Commons licence, unless indicated otherwise in a credit line to the material. If material is not 
included in the article's Creative Commons licence and your intended use is not permitted by statutory regulation or exceeds the permitted use, you will need to obtain permission directly from the copyright holder. To view a copy of this licence, visit http://creativecommons .org/licenses/by/4.0/.

\section{References}

1. Crane LJ (1970) Flow past a stretching plate. Z Angew Math Phys ZAMP 21:645-647

2. Khedr MEM, Chamkha AJ, Bayomi M (2009) MHD flow of a micropolar fluid past a stretched permeable surface with heat generation or absroption. Nonlinear Anal Model Control 14(1):27-40

3. Magyari E, Chamkha AJ (2010) Combined effect of heat generation or absorption and first-order chemical reaction on micropolar fluid flows over a uniformly stretched permeable surface: the full analytical solution. Int J ThermSci 49:1821-1828

4. Seyadi SH, Saray BN, Chamkha AJ (2020) Heat and mass transfer investigation of MHD Eyring-Powell flow in a stretching channel with chemical reactions. Phys A 544:124109

5. DamsehRebhi A, Al-Odat MQ, Chamkha AJ, ShannakBenbella A (2009) Combined effect of heat generation or absorption and first-order chemical reaction on micropolar fluid flows over a uniformly stretched permeable surface. Int J ThermSci 48:1658-1663

6. Takhar HS, Chamkha AJ (2001) Unsteady three-dimensional MHD-boundary-layer flow due to the impulsive motion of a stretching surface. ActaMech 146:59-71

7. Mukhopadhyay S (2013) Slip effects on MHD boundary layer flow over an exponentially stretching sheet with suction/blowing and thermal radiation. Ain Shams Eng J 4(3):485-491

8. Hsiao K-L (2017) Micropolarnanofluid flow with MHD and viscous dissipation effects towards a stretching sheet with multimedia feature. Int J Heat Mass Transf 112:983-990

9. Hsiao K-L (2016) Stagnation electrical MHD nanofluid mixed convection with slip boundary on a stretching sheet. ApplThermEng 98:850-861

10. Choi SUS (1995) Enhancing thermal conductivity of fluids with nano-particles. ASME IntMechEng 66:99-105

11. Buongiorno J (2006) Convective transport in nanofluids. ASME $J$ Heat Transf 128:240-250

12. Daniel YS, Aziz ZA, Ismail Z, Bahar A, Salah F (2019) Stratified electromagnetohydrodynamic flow of nanofluid supporting convective role. Korean J ChemEng 36(7):1021-1032

13. Hayat T, Asad S, Mustafa M, Alsaedi A (2014) Boundary layer flow of Carreau fluid over a convectively heated stretching sheet. Appled Math Comput 246:12-22

14. Machireddy GR, Naramgari S (2018) Heat and mass transfer in radiative MHD Carreau fluid with cross diffusion. Ain Shams Eng J 9:1189-1204

15. Ramadevi B, Sugunamma V, Anantha KK, Ramana Reddy JV (2019) MHD flow of Carreau fluid over a variable thickness melting surface subject to Cattaneo-Christov heat flux. Multidiscip Model Mater Struct 15(1):2-25

16. Khan M, Malik MY, Salahuddin T, Khan I (2017) Numerical modeling of Carreau fluid due to variable thicked surface. Results Phys 7:2384-2390
17. Farooq M, UlainAnzar Q, Hayat T, ljaz Khan M, Anjum A (2017) Local similar solution of MHD stagnation point flow in Carreau fluid over a non-linear stretched surface with double stratified medium. Results Phys 7:3078-3089

18. Khan I, Ullah S, Malik MY, Hussain A (2018) Numerical analysis of MHD Carreau fluid flow over a stretching cylinder with homogeneous-heterogeneous reaction. Results Phys 9:1141-1147

19. Eid MR, Mahny KL, Muhammad T, Sheikholeslami M (2018) Numerical treatment for Carreaunanofluid flow over a porous nonlinear stretching surface. Results Phys 8:1185-1193

20. Kumar RK, Kumar GV, Raju CS, Shehzad SA, Varma SV (2018) Analysis of Arrhenius activation energy in magnetohydrodynamicCarreau fluid flow through improved theory of heat diffusion and binary chemical reaction. J PhysCommun 2(3):035004

21. Sulochana C, Ashwinkumar GP, Sandeep N (2016) Transpiration effect on stagnation-point flow of a Carreaunanofluid in the presence of thermophoresis and Brownian motion. Alex Eng J 55:1151-1157

22. Kala BS, Rawat MS, Rawat N, Kumar A (2019) Numerical analysis of non-Darcy MHD flow of a Carreau fluid over an exponentially stretching/shrinking sheet in a porous medium. Int J Sci Res Math Stat Sci 6(2):295-303

23. Mamatha SU, Raju CSK, Makinde OD (2017) Effect of convective boundary conditions on MHD carreau dusty fluid over a stretching sheet with heat source. DeffectDiffus Forum 377:233-241

24. Waqas M, ljaz Khan M, Hayat T, Alsaedi A (2017) Numerical simulation for magneto Carreaunanofluid model with thermal radiation: a revised model. Comput Methods ApplMechEng 324:640-653

25. Khan M, Azam M (2017) Unsteady heat and mass transfer mechanisms in MHD Carreaunanofluid flow. J MolLiq 225:554-562

26. Gorla RSR, Chamkha A (2011) Natural convective boundary layer flow over a nonisothermal vertical plate embedded in a porous medium saturated with a nanofluid. NanoscaleMicroscaleThermophysEng 15:81-94

27. Chamkha AJ, Aly AM (2011) MHD Free convection flow of a nanofluid past a vertical plate in the presence of Heat generation or absorption effects. ChemEngCommun 198:425-441

28. Chamkha AJ, Aly AM, Mansour MA (2010) Similarity solution for unsteady heat and mass transfer from a stretching surface embedded in a porous medium with suction/injection and chemical reaction effects. ChemEngCommun 197:846-858

29. Hsiao K-L (2017) To promote radiation electrical MHD activation energy thermal extrusion manufacturing system efficiency by using Carreau-nanofluid with parameters control method. Energy 130:486-499

30. Irfan M, Khan WA, Khan M, MudassarGulzar M (2019) Influence of Arrhenius activation energy in chemically reactive radiative flow of 3D Carreaunanofluid with nonlinear mixed convection. J PhysChem Solids 125:141-152

31. Irfan M, Khan M, Khan WA, Ahmad L (2019) Influence of binary chemical reaction with Arrhenius activation energy in MHD nonlinear radiative flow of unsteady Carreaunanofluid: dual solutions. ApplPhys A 125:179

32. Alsaadi FE, Hayat T, Khan MI, Alsaadi FE (2020) heat transport and entropy optimization in flow of magneto-Williamson nanomaterial with Arrhenius activation energy. Comput Methods Programs Biomed 183:105051

Publisher's Note Springer Nature remains neutral with regard to jurisdictional claims in published maps and institutional affiliations. 